\title{
Decay of Metastable State with Account of Agglomeration and Relaxation Processes
}

\author{
Victor Kurasov \\ Saint Petersburg State University, Universitetskaya Embankment 7/9, Saint Petersburg 199034, Russia \\ Correspondence should be addressed to Victor Kurasov; victor_kurasov@yahoo.com
}

Received 27 July 2015; Revised 5 November 2015; Accepted 8 November 2015

Academic Editor: Leonardo Palmisano

Copyright (C) 2016 Victor Kurasov. This is an open access article distributed under the Creative Commons Attribution License, which permits unrestricted use, distribution, and reproduction in any medium, provided the original work is properly cited.

Theoretical description of the metastable phase decay kinetics in the presence of specific connections between the embryos of small sizes has been given. The theory of the decay kinetics in the presence of relaxation processes is constructed in analytical manner. The $m$-mers nucleation is investigated and the global kinetics of decay is also constructed in this case analytically.

\section{Introduction}

The first-order phase transition kinetics is actively investigated since the publication of the pioneer papers by Wilson [1-3] concerning the famous chamber which became the main tool in investigations of the microworld. The range of applications of the first-order phase transition kinetics inevitably grows since the phenomena concerning the selforganization [4,5] and aggregation [6] become the field of application of ideas lying in the base of the mentioned kinetics. The word "nucleation" has nothing in common with nuclear phenomena but comes from the evident fact that the embryos of a new phase are rather compact objects and can be treated as some nucleus of a new phase. The main content of the theory which predicts the rate of appearance of the embryos of a new phase was published in several papers in the 1930s and 1940s which forms the classical theory of nucleation. The classical theory of nucleation $[7,8]$ gives a complete theoretical description but the problem is that there exists no correspondence between the theoretical predictions and experimental results.

The first theoretical investigation on the problem of the embryos (droplet) formation belong to W. Thomson (1870), who got the work of formation of the critical embryo and the size of the critical embryo. However, Laplace and Young were able to do this after their invention of the additional pressure under the curved surface. Certainly, the true meaning of the formulas arises only with the theoretical investigations by Gibbs (1877).
Historically the classical nucleation theory is associated with the following four papers [9-12] although many other contributions (see, e.g., [13], where for the first time was stressed that the preexponential kinetic factor has to account collisions of a vapor molecule with cluster) are rather important in creation of the nucleation theory.

After the creation of the classical nucleation theory the most radical reconsideration was proposed by Lothe and Pound [14] who suggested including into the free energy of a cluster the translational and rotational degrees of freedom. This point of view leads to the reconsideration of the rate of nucleation in dozen of orders of magnitudes. In some cases it brings the theory to the coincidence with experiment; in some cases the situation is the opposite one. The point of view of Lothe and Pound was reconsidered in $[15,16]$ and the effect in comparison with the classical nucleation theory became radically smaller. Here it is necessary to stress that the arguments of additional consideration of rotational and translational degrees of freedom for an embryo are rather doubtful.

Other approaches to improve the classical nucleation theory do not suggest such radical improvements. One can mention here the attempts to account the partial pressure of the cluster which is treated as a molecule $[17,18]$. The effect of this idea is not too big in comparison with the effect of LothePounds' reconsideration.

The next approach is the so-called kinetic theory of nucleation suggested in $[19,20]$. The long derivations in order 
to solve the chain of kinetic equations finally led to the practically known results except the requirement to take into account the term corresponding to the surface tension of a cluster with one molecule which can not be treated formally in this sense but is certainly rather small correction factor.

The Dillmann-Meier theory of nucleation is a rather model version which can be treated as some postulate on the surface tension of a curved surface together with requirements of thermodynamic consistency and some other minor improvements [21,22]. Certainly the theoretical ground of this approach is rather doubtful although the ideas are physically attractive.

The famous paper of Cahn and Hillard [23] is very beautiful theoretically but the jumps of the density between phases can be hardly described only by the first nontrivial term in the gradient decomposition. This makes the range of application of this approach rather narrow, namely, in the vicinity of a critical point. But in this vicinity one can hardly consider the embryo as a closed object. So, the use of the mentioned approach is rather restricted.

One can not find any mathematical gaps in the classical theory of nucleation. It means that some physical assumptions of the model are not too appropriate since the mathematical frames of theoretical construction are certainly correct.

One of the possible sources of the deviation of the theoretical predictions from the experimental results is the existence of the clusters (nonmonomers) with a relatively small free energy which actively changes the picture of the nucleation process. Nowadays effect is known under the name of prenucleation [24], although some of such clusters can belong to the blind channels which can not lead to the big supercritical clusters [25]; they are not nucleation centers. Nevertheless such terminology exists.

The last example belongs to biological systems. The complex behavior of the nucleation kinetics in biological systems is not a surprising fact since the complex structure of biological molecules is well known. More intriguing is the prenucleation importance in calcium containing systems [26]. The further analysis of the prenucleation phenomena [27] shows the possibility of many paths through a nucleation barrier to a supercritical zone. The nucleation processes through the different channels can even compete [28]. Unfortunately the experimental observations are rarely accompanied by the weighted theoretical constructions.

An evident intention to solve the appearing problems is to use computer calculations. Unfortunately it is impossible to check all suppositions of the theory by computer simulation since the nucleation process is the principally collective phenomenon and the true simulation has to consider billions of particles with precise numerical integration because the effect of nucleation is hidden in excesses from the phase coexistence line.

Ordinarily the attempts to improve the theory are aimed at getting the more reliable expression for the free energy of the critical embryo. There is no doubt that the critical embryo is the central object of the nucleation theory consideration but the constructions of the classical nucleation theory approach are valid only under the "good" behavior of the free energies of embryos of other sizes and kinetic coefficients of ejection and absorption of the molecules for the embryos far from the critical one.

The "good" behavior means that there are no peculiarities in behavior of kinetic coefficients and no local extremal points of the free energy of classically noncritical embryos. Meanwhile there is absolutely no insurance that the mentioned "good" behavior really takes place. The experimental information is rather poor, kinetic coefficients are mainly measured on the basis of the growth rate [29], and this characteristic is measured with such an accuracy that one can put a question about the excesses in the growth rate [30]. The measurements of the growth rates can give only the difference between the adsorption and ejection coefficients but not exactly the values of these coefficients.

One can note that in the presence of the prenucleation centers the reconsideration of the rate of the stationary nucleation can be a nontrivial problem only in situations when the embryos have rather complex structure coming from various structural components (see, e.g., [25]). The methods to construct the stationary rate of nucleation are rather well known although one can find many interesting subjects in this question also.

Here we will focus on the kinetics of the global nucleation, that is, on the description of the global evolution of the system starting from the appearance of metastability up to the end of the active formation of the embryos of a new phase which grow irreversibly until the Ostwald ripening. The last stages of the phase transition including the coagulation [31] and Lifshic-Slezov asymptote $[32,33]$ are not the subject of this analysis because we are interested in the total number of supercritical embryos and formation of their sizes spectrum. The total number of embryos and their size spectrum are the main results of the phase transformation and later the coagulation and mutual consumption (it is better to use, namely, this terminology because the ordinary used word "coalescence" implies absolutely another physical process) will only deform the size spectrum and the total number of supercritical embryos.

To describe the global nucleation kinetics it is necessary to put the initial conditions. This requirement seems to be quite natural. We suppose that at the initial moment of time there exists in the system a metastable phase without a new phase and the process of nucleation begins. Otherwise we can define the initial moment as the last moment before appearance of the first traces of a new phase. No further external action on the system is implied. This type of the initial and boundary conditions is quite natural and has the name of the decay of the metastable state.

The situation of decay without peculiarities in behavior of the free energy of a small cluster was considered in [34] where the complete theory was constructed. It is shown that the evolution of the system can be described by the substance balance equation in some special form. For our purposes to describe the kinetics in the situation where one size of clusters is extracted by a very small free energy the analogy with heterogeneous nucleation will be very useful. In heterogeneous nucleation the kinetics of the process is governed not only by the fall of the supersaturation but also by 
the exhaustion of heterogeneous centers which become the centers of the droplets. Here the theory was given in 1989 [35] but the gap in the theory was filled only in 1993 [36].

The peculiarities in connections between the small clusters in a mother phase can cause very specific behavior of the global nucleation kinetics. Although already many important cases were considered there exist enough important situations necessary to be described theoretically. Below we will give the theoretical description of some of them.

\section{Weak Relaxation of Seeds in Monomer Nucleation}

The first important case is the situation when in the monomer nucleation there exists a weak connection between the region (in the scale of sizes) of monomers and the region of $m$-mers and it is necessary to take into account not only the consumption of $m$-mers by the supercritical embryos (every embryo has to consume one $m$-mer to start the life) but also the weak source of monomers being transformed into $m$-mers. This source changes the kinetics of the process.

Here it is very useful to use the analogy with heterogeneous nucleation. Really, the $m$-mers can be considered as some objects analogous to heterogeneous centers. But the situation with the relaxation of heterogeneous centers has not been considered. So, there exists a nontrivial mathematical content of the constructions presented below.

We introduce the value of $\theta$ as

$$
\theta=\frac{n[m, t]}{n[m, t=0]} .
$$

This value can be less or greater than 1 . Here $n[m, t]$ is the number of embryos with $m$ molecules at the time moment $t$.

One can formulate the system of equations for unknown functions $g$ and $\theta$ and after some scaling get

$$
\begin{gathered}
\frac{d \theta}{d t}=-j\left(\theta-\theta_{\lim }\right)-T \theta[t] \exp (-g[t]) \\
g[t]=\int_{0}^{t}\left(t-t^{\prime}\right)^{3} \theta\left[t^{\prime}\right] \exp \left(-g\left[t^{\prime}\right]\right) d t^{\prime} .
\end{gathered}
$$

Here $T$ is some positive parameter which can not be avoided. The positive parameter $j$ shows the intensity of the relaxation process. The value $\theta_{\lim }$ is the equilibrium value. It is also a parameter.

Now we will clarify the meaning of two unknown functions $g$ and $\theta$ of the time $t$. The function $g$ is the relative number of the molecules in a new phase. The function $\theta$ is the relative number of the free (unoccupied by the supercritical embryos) heterogeneous centers.

Ordinarily the solution of the system of condensation equations (analogous to (2)-(3)) is given by iterations (see [37]). The behavior of the spectrum of sizes

$$
f[t]=\theta[t] \exp (-g[t])
$$

is rather complex even already in the first iterations which are constructed in a way to simplify the form of the size spectrum. This fact means that in our case one can not invent too "intelligent" iterations. Namely, the presentation of (3) in the already integrated form seems to produce too complex iteration procedure.

In investigation of the nucleation process with the absence of the relaxation process (see [37]) the following chains of inequalities,

$$
\begin{aligned}
& \theta_{(1)}<\theta_{(3)}<\cdots<\theta<\cdots<\theta_{(2)} \\
& g_{(0)}<g_{(2)}<\cdots<g<\cdots<g_{(3)}<g_{(1)},
\end{aligned}
$$

were formulated. This chain is very productive for the accuracy estimates which lead to the guaranteed accuracy of the obtained approximations.

To conserve these chains one can propose the following recurrent scheme:

$$
\begin{gathered}
\frac{d \theta_{(i+1)}}{d t}=-j\left(\theta_{(i)}-\theta_{\lim }\right)-T \theta_{(i)}[t] \exp \left(-g_{(i)}[t]\right) \\
g_{(i+1)}[t]=\int_{0}^{t}\left(t-t^{\prime}\right)^{3} \theta_{(i)}\left[t^{\prime}\right] \exp \left(-g_{(i)}\left[t^{\prime}\right]\right) d t^{\prime}
\end{gathered}
$$

with initial condition

$$
\theta[t=0]=1
$$

and initial approximations

$$
\theta_{(0)}=\theta_{\text {lim }}
$$

or

$$
\begin{aligned}
\theta_{(0)}[t] & =\theta[t=0], \\
g_{(0)} & =0
\end{aligned}
$$

or the recurrent scheme

$$
\begin{aligned}
\frac{d \theta_{(i+1)}}{d t}= & -j\left(\theta_{(i+1)}-\theta_{\lim }\right) \\
& -T \theta_{(i+1)}[t] \exp \left(-g_{(i)}[t]\right) \\
g_{(i+1)}[t]= & \int_{0}^{t}\left(t-t^{\prime}\right)^{3} \theta_{(i)}\left[t^{\prime}\right] \exp \left(-g_{(i)}\left[t^{\prime}\right]\right) d t^{\prime}
\end{aligned}
$$

with the same initial condition and the same initial approximations. The third scheme is

$$
\begin{gathered}
\frac{d \theta_{(i+1)}}{d t}=-j\left(\theta_{(i)}-\theta_{\lim }\right)-T \theta_{(i+1)}[t] \exp \left(-g_{(i)}[t]\right) \\
g_{(i+1)}[t]=\int_{0}^{t}\left(t-t^{\prime}\right)^{3} \theta_{(i)}\left[t^{\prime}\right] \exp \left(-g_{(i)}\left[t^{\prime}\right]\right) d t^{\prime}
\end{gathered}
$$

with the same initial approximations and the same initial condition.

We have to choose what type of iterations is the best one.

The first procedure is absolutely correct and it can not lead to divergence at some step of iterations. The second and the third schemes require additional regularization. One has to put an additional restriction. 
The Nucleation Process Stops When $\theta$ Attains Zero. This restriction is necessary to avoid the negative rate of nucleation and the divergence of iterations.

The account of the relaxation process in the nucleation kinetics is a hard problem. Already the first iteration from those where the effect of the relaxation term can be seen in the behavior of the supersaturation can not be calculated analytically.

To fulfil analytical calculations we have to fulfil an additional simplification based on the property of the avalanche consumption.

The mentioned property can be formulated as follows.

Under Any Behavior of $\theta>0$ the Relative Growth of $g$ Occurs Faster Than $\left(t-t_{0}\right)^{3}$. One can prove this property analytically. Actually, this property is evident since we take into account that $g=\int_{0}^{t}\left(t-t^{\prime}\right)^{3} \theta\left[t^{\prime}\right] \exp \left(-g\left[t^{\prime}\right]\right) d t^{\prime}$ and $\theta>0$.

On the basis of this property we see that the "pseudohomogeneous spectrum of sizes" (the spectrum of sizes which would be formed if there would be no action of the number of free seeds on the nucleation process)

$$
f_{\text {hom }} \sim \exp (-g)
$$

will be truncated in a manner sharper than

$$
\exp \left(-\left(\frac{t}{t_{\text {cut }}}\right)^{3}\right)
$$

where $t_{\text {cut }}$ is the characteristic time. Then one can speak about the cut-off type of the pseudo-homogeneous spectrum of sizes.

Now one can see the qualitative behavior of $\theta$. At first $\theta$ relaxes to the value

$$
\theta_{n}=\frac{j}{j+T} \theta_{\lim }
$$

with characteristic time

$$
t_{\text {rel } n}=(j+T)^{-1} .
$$

This relaxation can lead to the increase of $\theta$ when

$$
\theta[t=0]<\theta_{n}
$$

or to the decrease of $\theta$ in the opposite situation.

At the moment $t_{\text {cut }}$ this relaxation is changed by the relaxation of $\theta$ to $\theta_{\lim }$ with the characteristic time $t_{\text {rel }}=j^{-1}$.

The question which we are interested in is whether the change of $\theta$ can destroy the cut-off type of the real spectrum of sizes $\theta \exp (-g)$ (earlier the cut-off type was shown for $\exp (-g))$

The destruction of the cut-off type of $\theta \exp (-g)$ can take place only under the sharp relative increase of $\theta$. So, we have to estimate $(d \theta / d t) / \theta$. Since

$$
\begin{aligned}
\frac{d \theta}{d t} & <j \theta_{\lim } \\
\theta & >\frac{j}{j+T} \theta_{\lim }
\end{aligned}
$$

we see that

$$
\frac{d \theta / d t}{\theta}<j+T
$$

When $T \gg j$ one simply can not see the relaxation. The picture in this case is the picture described earlier (see [37]) plus some small perturbation. Then having excluded this situation we can rewrite the last inequality as

$$
\frac{d \theta / d t}{\theta}<(3 \div 4) j
$$

Now we see that the danger of destruction of the cut-off form of the size spectrum can appear only at big values of $j$. Actually it has to be

$$
j \gg t_{\text {cut }}^{-1}
$$

If $t_{\text {cut }} \ll T^{-1}$ then there is no exhaustion of seeds. So, the actual situation is $t_{\text {cut }} \geq T^{-1}$ and then we see that

$$
j \gg T \text {. }
$$

Here $\theta_{\text {lim }}$ is close to $\theta_{n}$ and then $t_{\text {cut }}$ based on $\theta_{\text {lim }}$ is close to $\theta$ based on $\theta_{n}$. This means that the cut-off behavior is not broken here. So, the property of the cut-off takes place in all situations.

The property of the cut-off allows using the approximation $\exp (-g)=1$ for $t<t_{\text {cut }}$ and $\exp (-g)=0$ for $t>t_{\text {cut }}$. The moment $t_{\text {cut }}$ can be determined as the root of equation

$$
g\left(t_{\text {cut }}\right)=1 \text {. }
$$

Then for $t<t_{\text {cut }}$

$$
\begin{gathered}
\frac{d \theta}{d t}=-j\left(\theta-\theta_{\lim }\right)-T \theta[t] \\
g[t]=\int_{0}^{t}\left(t-t^{\prime}\right)^{3} \theta\left[t^{\prime}\right] d t^{\prime}
\end{gathered}
$$

and one can explicitly calculate

$$
\theta[t]=\theta_{n}-\left(\theta_{n}-\theta[t=0]\right) \exp (-(j+T) t) .
$$

The value of $g$ can be presented as

$$
\begin{aligned}
g & =\int_{0}^{t}\left(t-t^{\prime}\right)^{3} \\
& \cdot\left(\theta_{n}-\left(\theta_{n}-\theta[t=0]\right) \exp \left(-(j+T) t^{\prime}\right)\right) d t^{\prime}
\end{aligned}
$$

and can be easily found analytically which allows getting $t_{\text {cut }}$ as the solution of an ordinary algebraic equation.

Equation (22) can be easily solved since we know the estimates $\theta_{n} z^{4} / 4$ and $\theta[0] z^{4} / 4$ for the behavior of $g$ which leads to the estimates for the roots. What estimate is the above estimate and what the below estimate is are determined by the sign of $\theta_{\lim }-\theta[0]$.

The total number of droplets in the renormalized units can be found as

$$
N=A \int_{0}^{t_{\text {cut }}} 1 d t=A t_{\text {cut }}
$$


where $A$ is the initial amplitude (in renormalized values it equals 1 ) or as

$$
N=A \int_{0}^{t_{\mathrm{cut}}} \exp (-g[t]) \theta[t] d t
$$

where for $g$ and for $\theta$ one can take the mentioned approximations. The linearization of appearing exponents is also approximately suitable and leads to the presence of the result in elementary functions. One has to note that the first recipe seems to be more rough than the second one but it gives better results. The reason is the effective compensation of decrease of the intensity of appearance of new embryos before $t_{\text {cut }}$ by the existence of the tail after $t_{\text {cut }}$. The refinement of equation for $t_{\text {cut }}$ (it is better to use $g\left[t_{\text {cut }}\right]=\ln 2$ ) can make the first recipe very accurate. Certainly the further refinement with the help of the perturbation technique can be fulfilled.

Even the linear approximation

$$
\theta=\theta[0]+(j+T)\left(\theta_{n}-\theta[0]\right) t
$$

truncated at the crossing point with $\theta_{n}$, that is, at $(j+T)^{-1}$, and prolonged further as $\theta_{n}$ gives a rather good approximation for the characteristics of the process. This approximation ensures the pure algebraic equation of the fifth power for $t_{\text {cut }}$. It is a really suitable approximation since this approximation has the maximal deviation as $(j+T)^{-1}$ which is relatively small $(\sim \exp (-1))$ in comparison with initial deviation of $\theta$ from $\theta_{n}$. Then the difference in integrals staying in (22) is less than two times which gives the difference for the duration of the intensive nucleation period in $2^{1 / 4} \approx 1.2$ times (in reality the difference in duration is much more small). The last approximation brings equation on root to the explicit formulas.

This completes the analytical description of the situation of the decay in the system with the relaxation process for the centers of nucleation.

\section{Accumulation of Several Seeds in the Monomer Nucleation}

The second important task is to consider the situation when it is necessary to spend $q m$-mers to form the supercritical embryo and the rate of nucleation is proportional to the number of $m$-mers in power $q$ :

$$
J \sim n[m]^{q} .
$$

It is interesting to investigate this situation and to see the transition to the case when $q$ is great enough. Certainly the probability

$$
P \sim n[m]^{q}
$$

transfers into

$$
P \sim \exp (-\mu q)
$$

with a chemical potential

$$
\mu=-\ln (n[m])
$$

corresponding to the chemical potential of ideal gas.

The equation

$$
\frac{d n[m]}{d t}=n[m]^{q} f[t]
$$

with some known function $f[t]$ can be written in the following integral form:

$$
n[m]=\frac{n[m, t=0]}{\left((q-1) \int_{0}^{t} f\left[t^{\prime}\right] d t^{\prime}+1\right)^{1 /(q-1)}} .
$$

Now we write the expression for the rate of nucleation in the generalized Clausius-Clapeyron integrated form as

$$
J=J_{0} \theta^{q} \exp \left(-\frac{\Gamma\left(\zeta-\Phi_{*}\right)}{\Phi_{*}}\right),
$$

where

$$
\Gamma=\left.\frac{\Phi_{*} d F_{c}}{d \zeta}\right|_{\zeta=\Phi_{*}}
$$

and $\Phi_{*}$ is the base of decomposition. Here $J_{0}$ is the initial value of the rate of nucleation and $\theta$ is the relative number of $m$-mers referred to initial value.

One can get

$$
\Gamma=\left(\frac{\Phi_{*}}{\left(\Phi_{*}+1\right)}\right) v_{1 c}
$$

where $v_{1 c}$ is the number of monomers in critical embryo. This number is connected with the total number of molecules $\nu_{c}$ in the critical embryo as

$$
v_{c}=v_{1 c}+q m
$$

After the scaling the system of evolution equations is the following one:

$$
\begin{aligned}
& g[z]=\int_{0}^{z}(z-x)^{3} \theta^{q}[x] \exp (-g[x]) d x \\
& \theta[z]=\left((q-1) A \int_{0}^{z} \exp (-g[x]) d x+1\right)^{-1 /(q-1)} .
\end{aligned}
$$

Here $A$ is the coefficient which remains after the scaling and can not be canceled.

We can formulate the iteration procedure as follows:

$$
\begin{aligned}
& g_{(i+1)}(z)=\int_{0}^{z}(z-x)^{3} \theta_{(i)}^{q}[x] \exp \left(-g_{(i)}[x]\right) d x \\
& \theta_{(i+1)}[z]
\end{aligned}
$$

$$
=\left((q-1) A \int_{0}^{z} \exp \left(-g_{(i)}[x]\right) d x+1\right)^{-1 /(q-1)} .
$$


The separation of the spectrum in $g$ and $\theta$ which seems to be natural from the physical point of view is quite unusual from the formal point of view when the unique equation

$$
\begin{aligned}
& g[z]=\int_{0}^{z}(z-x)^{3} \\
& \cdot\left((q-1) A \int_{0}^{x} \exp \left(-g\left[x^{\prime}\right]\right) d x^{\prime}+1\right)^{-q /(q-1)} \\
& \cdot \exp (-g[x]) d x
\end{aligned}
$$

is considered. Then it is more natural to construct iterations as

$$
\begin{aligned}
& g_{(i+1)}[z]=\int_{0}^{z}(z-x)^{3} \\
& \cdot\left((q-1) A \int_{0}^{x} \exp \left(-g_{(i)}\left[x^{\prime}\right]\right) d x^{\prime}+1\right)^{-q /(q-1)} \\
& \cdot \exp \left(-g_{(i)}[x]\right) d x
\end{aligned}
$$

but this way does not correspond to appearance of the above and below estimates although it is possible to prove that these iterations with the initial approximation

$$
g_{(0)}=0
$$

converge to solution.

The procedure for $\theta$ and $g$ as the separate functions which was formulated above will lead under the initial approximations

$$
\begin{aligned}
& \theta_{(0)}=1, \\
& g_{(0)}=0
\end{aligned}
$$

to the chains of inequalities

$$
\begin{aligned}
& g_{(0)}<g_{(2)}<\cdots<g<\cdots<g_{(3)}<g_{(1)} \\
& \theta_{(1)}<\theta_{(3)}<\cdots<\theta<\cdots<\theta_{(2)}<\theta_{(0)} .
\end{aligned}
$$

Parameter $A$ is considered here as constant value for all iterations and the concrete value of $A$ will be determined on the basis of the last (precise) iteration.

It is useful to calculate the total number of supercritical embryos as

$$
N_{\text {tot }} \sim \int_{0}^{\infty} \theta^{q}[x] \exp (-g[x]) d x .
$$

On the basis of iteration procedure one can calculate

$$
N_{\text {tot }(i+1)} \sim \int_{0}^{\infty} \theta_{(i)}^{q}[x] \exp \left(-g_{(i)}[x]\right) d x .
$$

The calculation of iterations gives

$$
\begin{aligned}
& g_{(0)}=\frac{z^{4}}{4} \\
& \theta_{(1)}=((q-1) A z+1)^{-1 /(q-1)} .
\end{aligned}
$$

Then

$$
N_{\text {tot(2) }}=\int_{0}^{\infty}((q-1) A z+1)^{-q /(q-1)} \exp \left(-\frac{z^{4}}{4}\right) d z
$$

and this can be expressed through the hypergeometric function

$$
\begin{aligned}
& N_{\text {tot }(2)}=2^{(-9 / 2+2(q /(q-1)))}(q+A)^{-q /(q-1)}\left(\frac{1}{3}\right. \\
& \cdot 2^{-(5 / 2)(q /(q-1))+1 / 2} \pi^{4}(q+A)^{3}\left(\frac{1}{q+A}\right)^{-q /(q-1)} H\left(\left[\frac{3}{4}\right.\right. \\
& \left.+\frac{1}{4} \frac{q}{q-1}, 1+\frac{1}{4} \frac{q}{q-1}, \frac{5}{4}+\frac{1}{4} \frac{q}{q-1}\right],\left[\frac{3}{2}, \frac{7}{4}, \frac{5}{4}\right], \\
& \left.-\frac{1}{4}(q+A)^{4}\right) \\
& \cdot \frac{\sec ((1 / 4) \pi(q /(q-1))) \Gamma(3+q /(q-1))}{\Gamma(3 / 2+(1 / 4)(q /(q-1)))} \\
& -2^{(1-(5 / 2)(q /(q-1)))} \pi^{4}(q+A)^{2}\left(\frac{1}{q+A}\right)^{-q /(q-1)} H\left(\left[\frac{3}{4}\right.\right. \\
& \left.+\frac{1}{4} \frac{q}{q-1}, 1+\frac{1}{4} \frac{q}{q-1}, \frac{1}{2}+\frac{1}{4} \frac{q}{q-1}\right],\left[\frac{3}{2}, \frac{3}{4}, \frac{5}{4}\right], \\
& \left.-\frac{1}{4}(q+A)^{4}\right) \\
& \cdot \frac{\csc ((1 / 4) \pi+(1 / 4) \pi(q /(q-1))) \Gamma(2+q /(q-1))}{\Gamma(5 / 4+(1 / 4)(q /(q-1)))} \\
& +2^{(5 / 2-(5 / 2)(q /(q-1)))} \pi^{4}(q+A)\left(\frac{1}{q+A}\right)^{-q /(q-1)} H\left(\left[\frac{3}{4}\right.\right. \\
& \left.+\frac{1}{4} \frac{q}{q-1}, \frac{1}{4}+\frac{1}{4} \frac{q}{q-1}, \frac{1}{2}+\frac{1}{4} \frac{q}{q-1}\right],\left[\frac{3}{4}, \frac{5}{4}, \frac{1}{2}\right], \\
& \left.-\frac{1}{4}(q+A)^{4}\right) \\
& \cdot \frac{\csc ((1 / 4) \pi(q /(q-1))) \Gamma(1+q /(q-1))}{\Gamma(1+(1 / 4)(q /(q-1)))} \\
& +2^{(3-(5 / 2)(q /(q-1)))} \pi^{4}\left(\frac{1}{q+A}\right)^{-q /(q-1)} H\left(\left[\frac{1}{4} \frac{q}{q-1}, \frac{1}{4}\right.\right. \\
& \left.\left.+\frac{1}{4} \frac{q}{q-1}, \frac{1}{2}+\frac{1}{4} \frac{q}{q-1}\right],\left[\frac{3}{4}, \frac{1}{2}, \frac{1}{4}\right],-\frac{1}{4}(q+A)^{4}\right) \\
& \cdot \frac{\sec ((1 / 4) \pi+(1 / 4) \pi(q /(q-1))) \Gamma(q /(q-1))}{\Gamma(3 / 4+(1 / 4)(q /(q-1)))} \\
& +2^{(13 / 2-2(q /(q-1)))} \pi^{3}\left(-\frac{1}{2}+\frac{1}{4} \frac{q}{q-1}\right)(q+A)
\end{aligned}
$$




$$
\begin{aligned}
& \cdot H\left(\left[1, \frac{3}{4}, \frac{1}{2}, \frac{1}{4}\right],\left[\frac{5}{4}-\frac{1}{4} \frac{q}{q-1},-\frac{1}{4} \frac{q}{q-1}+\frac{1}{2}\right.\right. \\
& \left.\left.-\frac{1}{4} \frac{q}{q-1}+\frac{3}{4},-\frac{1}{4} \frac{q}{q-1}+1\right],-\frac{1}{4}(q+A)^{4}\right) \Gamma(-2 \\
& \left.\left.+\frac{q}{q-1}\right)\right)\left(\pi^{3} \Gamma\left(\frac{q}{q-1}\right)\right)^{-1},
\end{aligned}
$$

where $\Gamma$ is the gamma-function and $H$ is the hypergeometric function.

It is possible to show analytically that this expression is rather accurate. The error is less than one percent. Despite the known form it is rather difficult to perform calculations according to this expression and it is necessary to simplify the calculations.

Again we use the property of the avalanche consumption. Here one can show that $\theta$ is the decreasing (or at least not rapidly increasing) function of time (or of the size of the initial embryo). Then one can see the cut-off type of the function $\exp (-g)$ and the possibility of truncation of the function $\theta^{q} \exp (-g(z))$ at $t_{\text {cut }}$. The value of $t_{\text {cut }}$ can be chosen as the root of equation $g=1$. Then the spectrum of sizes

$$
f \sim \theta^{q}[x] \exp (-g[x])
$$

can be approximated as

$$
f \sim((q-1) A z+1)^{-q /(q-1)}
$$

for $z<z_{\text {cut }}$, where $z_{\text {cut }}$ is the size corresponding to $t_{\text {cut }}$. For other $z$ the spectrum is zero.

The number of embryos can be easily calculated:

$$
\begin{aligned}
N_{\text {tot }} & =\int_{0}^{z}((q-1) A x+1)^{-q /(q-1)} d x \\
& =-\frac{(A z q-A z+1)^{-1 /(q-1)}-1}{A} .
\end{aligned}
$$

This solves the formal problem of our investigation.

To show the consistency of the presented approach we have to investigate the inclusion of the $m$-mers consumption kinetics into the general nucleation. Here one can find at least two aspects of the problem. The first aspect is the input of $m$-mers in the overcoming of activation barrier and the corresponding input in the stationary rate of nucleation. The second aspect is the consumption of the $m$-mers by the nearcritical and precritical embryos (this is the first type) and by the supercritical embryos (the second type). The rate of consumption will be different and their manifestation in the global kinetics $s$ will be described by different ways.

To clarify the first aspect we rewrite the stationary rate of nucleation as

$$
J \sim \exp (-q \ln (n[m])) \exp \left(-F_{c}\right) .
$$

One has to stress that here $F_{c}$ is the free energy of the critical embryo formation on $q m$-mers. This implies that the work of collecting of $m$-mers is considered separately. This work in approximation of ideal gas of $m$-mers is

$$
F_{0}=-q \ln (n[m]) \text {. }
$$

At the same time we have to note that in the embryo containing $\nu_{c}$ molecules there are only $v_{c}-q m$ molecules coming from monomers. Then $F_{0}$ has to be written as

$$
F_{0}=-b\left(\nu_{c}-q m\right)+\gamma A,
$$

where $b$ is the excess of the chemical potential for monomers, $\gamma$ is the renormalized surface tension, and $A$ is the surface area of the embryo. It is clear that in the determination of the surface area it is necessary to account that the part of the volume inside the embryos surface is occupied by the molecules coming from $m$-mers. When the surface of the embryo simply surrounds the embryo one has to write

$$
A=4 \pi\left(\frac{3 v_{l}\left(v_{1 c}+q m\right)}{4 \pi}\right)^{2 / 3}
$$

where $v_{l}$ is the volume per one molecule in a liquid phase and $v_{1 c}$ is the number of molecules coming from monomers.

In the state of equilibrium the chemical potential of $m$ mer has to be equal to $m$ chemical potentials of monomers (here we neglect the surface term or consider the deformed chemical potential). So

$$
\frac{n[m]}{n[m, s=0]}=\exp (b m)
$$

and we see that the total free energy $\widehat{F}$ is

$$
\widehat{F}_{c}=F_{c}-q b m=-v_{c} b+\gamma A
$$

and the total free energy coincides with the classical expression.

One has to stress that the parameter $\Gamma$ in the previous simplification of the dependence of the free energy on supersaturation (or the dependence of the stationary rate of nucleation on the supersaturation) is the number of molecules coming from monomers multiplied on $\Phi_{*} /\left(\Phi_{*}+1\right)$; that is,

$$
\Gamma \longrightarrow \Gamma_{1}=\frac{\Phi_{*} \nu_{1 c}}{\left(\Phi_{*}+1\right)}
$$

$\Gamma_{1}$ is $\Phi_{*} d F_{c} /\left.d \zeta\right|_{\zeta=\Phi_{*}}$.

If we calculate the derivative of the total free energy $\widehat{F}_{c}$ we get

$$
\left.\Phi_{*} \frac{d \widehat{F}_{c}}{d \zeta}\right|_{\zeta=\Phi_{*}}=\Gamma_{1}+q m \frac{d b}{d \zeta}
$$

or

$$
\left.\Phi_{*} \frac{d \widehat{F}_{c}}{d \zeta}\right|_{\zeta=\Phi_{*}}=\Gamma_{1}+\Gamma_{m},
$$


where

$$
\Gamma_{m}=\left.\frac{\zeta q m}{(\zeta+1)}\right|_{\zeta=\Phi_{*}}
$$

As a result

$$
\begin{aligned}
\left.\Phi_{*} \frac{d \widehat{F}_{c}}{d \zeta}\right|_{\zeta=\Phi_{*}} & =\Gamma . \\
\Gamma & =\left.\frac{\zeta \nu_{c}}{(\zeta+1)}\right|_{\zeta=\Phi_{*}}
\end{aligned}
$$

We will call this property the separation of $\Gamma$. It takes place only in the first derivatives of the free energies and only because the surface term does not act on the derivative of the free energy. The surface term in the free energy only changes the number of molecules in the critical embryo and has no direct influence on derivative.

So, we see that in the free energy there is the direct correspondence between the $m$-mers picture and monomers picture.

One can propose another picture when the number of $m$ mers necessary to form the critical embryo is proportional to the number of molecules inside the critical embryo. In the enormously big critical embryos, namely, this mechanism has to be considered. Then the picture is equivalent to the binary nucleation with two vapors (the vapor of monomers and the vapor of $m$-mers).

The second aspect is connected with the mechanism of consumption. Here one has to confess that there are two principal mechanisms of the substance consumption. The first mechanism is "one embryo-fixed number of clusters (monomers or $m$-mers)." The second mechanism is "one embryogrowing number of clusters (monomers and $m$-mers) corresponding to the law of the regular growth."

Rigorously speaking we have to write for each type of active monomers two types of consumption and the balance equations. With the help of exponential approximations mentioned above one can unify these mechanisms in one law of growth. Ordinarily when the first mechanism is important the second is absent. When the second mechanism acts it means that there is enough clusters of such type to neglect the exhaustion of seeds. We will call this effect as the dominating preference of one mechanism.

Despite the mentioned dominating preference property the simultaneous existence of several mechanisms can not be excluded as a specific case. But it is clear that with the help of approaches developed here it is possible to construct the theoretical description of any process with different mechanisms of formation, different mechanisms of substance consumption, and different relaxation processes corresponding to bounds or connections between the resources of clusters of different sizes.

The different relations between the characteristic times of the mentioned processes make the processes of nucleation rather different. The size spectrums are very various also. To describe these situations one has to demonstrate the power of the approaches making the base of theoretical description.
In every situation the leading idea will be the avalanche consumption of metastability in different manifestations of this property.

\section{Main Results}

This paper contains at least two important derivations concerning the kinetics of decay with account of the relaxation processes and the kinetics of decay when the m-mers nucleation is important. Both cases are important and one can see that account of these processes radically changes the numerical values of parameters of the nucleations process. But one has to confess that the qualitative picture remains similar to the traditional case. The last does not mean that nothing in the form of the particles size distribution is changed. Contrarily, the form of the size spectrum will be absolutely another. But the property of the avalanche cut-off of the pseudo-homogeneous size spectrum remains. The last property was very productive in construction of the above presented procedure.

It would be interesting to analyze these situations in the case of the smooth external influence on the system which causes nucleation, that is, in the case of dynamic conditions. Also it would be interesting to see the influence of the relaxation processes in the overcoming of the near-critical region in the process of the binary nucleation. Certainly, these perspectives make the subjects of separate publications.

Beside the direct construction of the nucleation global kinetics description we managed to extract some important features of the effect of $m$-mers inclusion into the nucleation process. One can mention here the property of separation of $\Gamma$ and the correspondence between the monomers picture and $m$-mers picture. These properties are important enough to be mentioned in the general theory of nucleation.

\section{Conflict of Interests}

The author declares that there is no conflict of interests regarding the publication of this paper.

\section{References}

[1] C. T. R. Wilson, "Condensation of water vapour in the presence of dust-free air and other gases," Philosophical Transactions of the Royal Society A: Mathematical Physical and Engineering Sciences, vol. 189, pp. 265-307, 1897.

[2] C. T. R. Wilson, "On the condensation nuclei produced in gases by the action of roentgen rays, uranium rays, ultraviolet rays and other agents," Philosophical Transactions A, vol. 192, no. 9, article 403, 1899.

[3] C. T. R. Wilson, "On the comparative efficiency as condensation nuclei of positively and negatively charged ions," Philosophical Transactions of the Royal Society A: Containing Papers of a Mathematical or Physical Character, vol. 193, pp. 289-308, 1900.

[4] A. Benmouna, R. Benmouna, M. R. Bockstaller, and I. F. Hakem, "Self-organization schemes towards thermodynamic stable bulk heterojunction morphologies: a perspective on future fabrication strategies of polymer photovoltaic architectures," Advances in Physical Chemistry, vol. 2013, Article ID 948189, 8 pages, 2013. 
[5] Y. Kazzi, H. Awada, and M. Nardin, "Adhesion on nanoorganized multilayers: surface thermodynamics and local energy dissipation," Advances in Physical Chemistry, vol. 2010, Article ID 502709, 11 pages, 2010.

[6] A. I. Burshtein, "Contact and distant luminescence quenching in solutions," Advances in Physical Chemistry, vol. 2009, Article ID 214219, 34 pages, 2009.

[7] A. C. Zettlemoyer, Nucleation, Dekker, New York, NY, USA, 1969.

[8] F. F. Abraham, Homogeneous Nucleation Theory, Academic, New York, NY, USA, 1974.

[9] M. Volmer and A. Weber, "Keimbildung in übersättigten Gebilden," Zeitschrift für Physikalische Chemie, vol. 119, pp. 277301, 1925.

[10] R. Becker and W. Döring, "Kinetische behandlung der keimbildung in überättigten dämpfern," Annals of Physics, vol. 24, pp. 719-752, 1935.

[11] J. Frenkel, "A general theory of heterophase fluctuations and pretransition phenomena," The Journal of Chemical Physics, vol. 7, no. 7, pp. 538-547, 1939.

[12] J. B. Zeldovich, "On the theory of new phase formation; cavitation," Acta Physicochimica USSR, vol. 18, pp. 1-22, 1943.

[13] L. Farkas, "The velocity of nucleus formation in superheated vapors," Zeitschrift für Physikalische Chemie, vol. 125, pp. 236240, 1927.

[14] J. Lothe and G. M. Pound, "Reconsiderations of nucleation theory," The Journal of Chemical Physics, vol. 36, no. 8, article 2080, 1962.

[15] H. Reiss, J. L. Katz, and E. R. Cohen, "Translation-rotation paradox in the theory of nucleation," The Journal of Chemical Physics, vol. 48, no. 12, pp. 5553-5560, 1968.

[16] H. Reiss, "Treatment of droplike clusters by means of the classical phase integral in nucleation theory," Journal of Statistical Physics, vol. 2, no. 1, pp. 83-104, 1970.

[17] W. G. Courtney, "Remarks on homogeneous nucleation," The Journal of Chemical Physics, vol. 35, no. 6, pp. 2249-2250, 1961.

[18] M. Blander and J. L. Katz, "The thermodynamics of cluster formation in nucleation theory," Journal of Statistical Physics, vol. 4, no. 1, pp. 55-59, 1972.

[19] J. L. Katz and H. Weidersich, "Nucleation theory without Maxwell Demons," Journal of Colloid and Interface Science, vol. 61, no. 2, pp. 351-355, 1977.

[20] J. L. Katz and M. D. Donohue, "A kinetic approach to homogeneous nucleation theory," in Advances in Chemical Physics, I. Prigogine and S. A. Rice, Eds., vol. 40, chapter 3, pp. 137-155, Wiley, 1979.

[21] A. Dillmann and G. E. A. Meier, "Homogeneous nucleation of supersaturated vapors," Chemical Physics Letters, vol. 160, no. 1, pp. 71-74, 1989.

[22] A. Dillmann and G. E. A. Meier, "A refined droplet approach to the problem of homogeneous nucleation from the vapor phase," The Journal of Chemical Physics, vol. 94, no. 5, pp. 3872-3884, 1991.

[23] J. W. Cahn and J. E. Hillard, "Free energy of a nonuniform system. III. Nucleation in a two-component incompressible fluid," The Journal of Chemical Physics, vol. 31, no. 3, pp. 688-699, 1959.

[24] D. Gebauer and H. Cölfen, "Prenucleation clusters and nonclassical nucleation," Nano Today, vol. 6, no. 6, pp. 564-584, 2011.
[25] D. Sept and J. A. McCammon, "Thermodynamics and kinetics of actin filament nucleation," Biophysical Journal, vol. 81, no. 2, pp. 667-674, 2001.

[26] A. S. Myerson and B. L. Trout, "Nucleation from solution," Science, vol. 341, no. 6148, pp. 855-856, 2013.

[27] D. Zahn, "Thermodynamics and kinetics of prenucleation clusters, classical and non-classical nucleation," ChemPhysChem, vol. 16, no. 10, pp. 2069-2075, 2015.

[28] H. Deng, S. Wang, X. Wang et al., "Two competitive nucleation mechanisms of calcium carbonate biomineralization in response to surface functionality in low calcium ion concentration solution," Regenerative Biomaterials, vol. 2, no. 3, pp. 187195, 2015

[29] D. Kozma and G. Tóth, "Microscopic rate constants of crystal growth from molecular dynamic simulations combined with metadynamics," Advances in Physical Chemistry, vol. 2012, Article ID 135172, 10 pages, 2012.

[30] S. Fakruddin, C. Srinivasu, B. R. Venkateswara Rao, and K. Narendra, "Excess transport properties of binary mixtures of quinoline with xylenes at different temperatures," Advances in Physical Chemistry, vol. 2012, Article ID 324098, 9 pages, 2012.

[31] A. A. Lushnikov, "Evolution of coagulating systems," Journal of Colloid and Interface Science, vol. 45, no. 3, pp. 549-556, 1973.

[32] I. M. Lifshic and V. V. Slezov, "Kinetics of diffusive decomposition of supersaturated solid solutions," Soviet Physics-Journal of Experimental and Theoretical Physics, vol. 35, pp. 331-339, 1959.

[33] I. M. Lifshic and V. V. Slezov, "The kinetics of precipitation from supersaturated solid solutions," Journal of Physics and Chemistry of Solids, vol. 19, no. 1-2, pp. 35-50, 1961.

[34] F. M. Kuni and A. P. Grinin, "Kinetics of homogeneous condensationat the stage of formation of the main quantity of a new phase," Colloid Journal of the USSR, vol. 46, p. 460, 1984.

[35] F. M. Kuni, T. Y. Novozhilova, and I. A. Terentiev, "Kinetic equation of heterogenous condensation," Letters in Mathematical Physics, vol. 14, no. 2, pp. 161-167, 1987.

[36] V. Kurasov, "Decay of metastable multicomponentmixture," http://arxiv.org/abs/cond-mat/9310005.

[37] V. B. Kurasov, "Kinetics of heterogeneous condensation under dynamic conditions," Physical Review E, vol. 49, no. 5, pp. 3948$3955,1994$. 

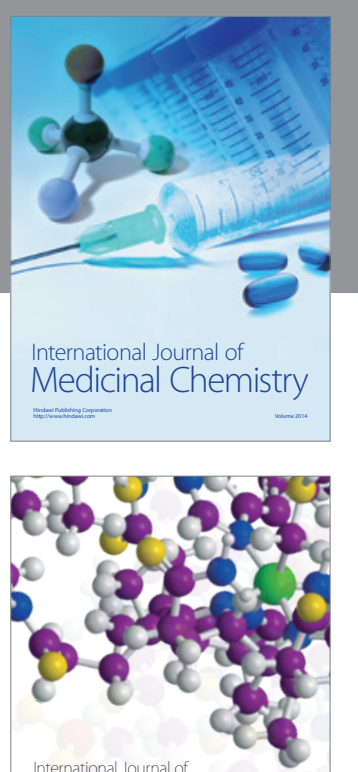

Carbohydrate Chemistry

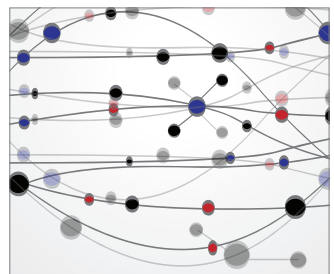

The Scientific World Journal
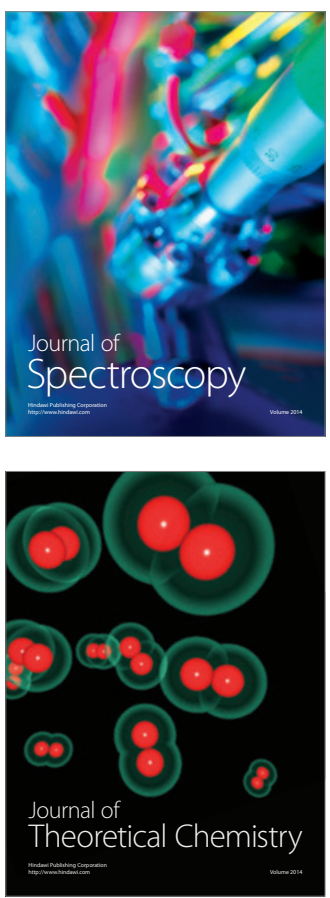
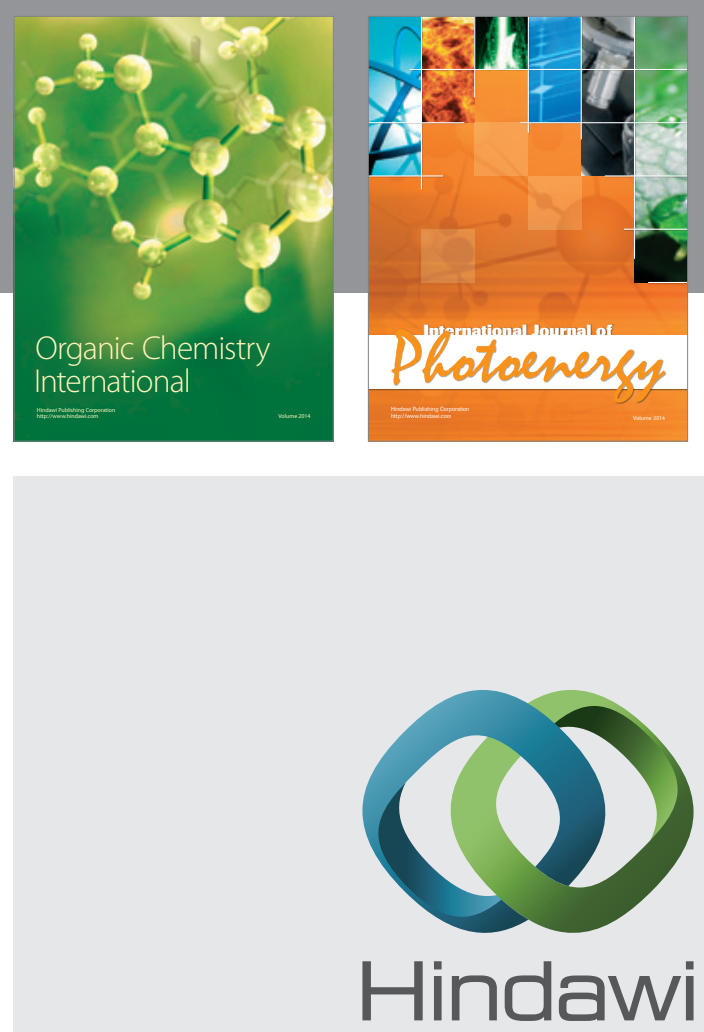

Submit your manuscripts at

http://www.hindawi.com

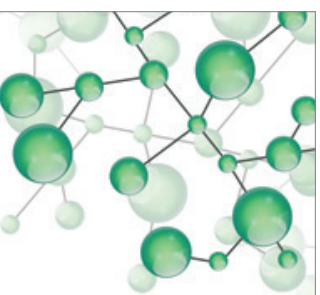

International Journal of

Inorganic Chemistry

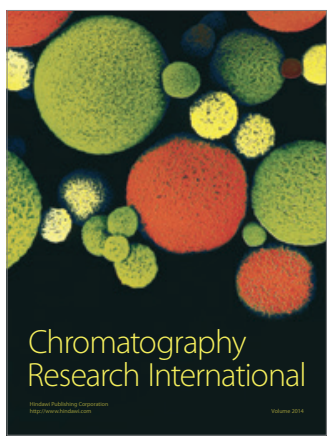

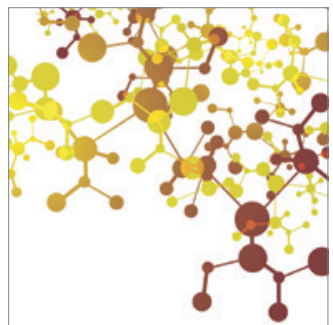

Applied Chemistry
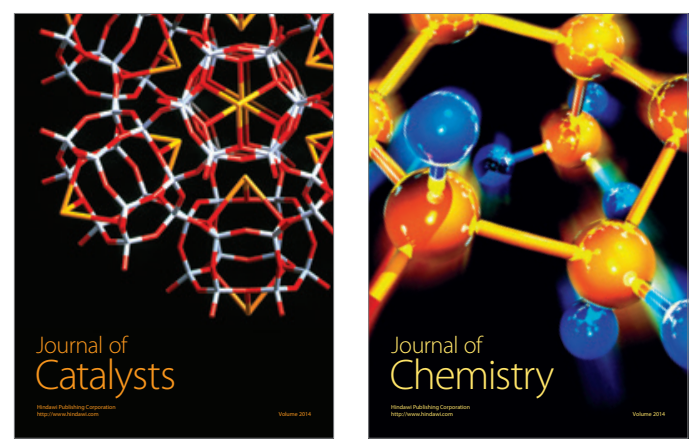
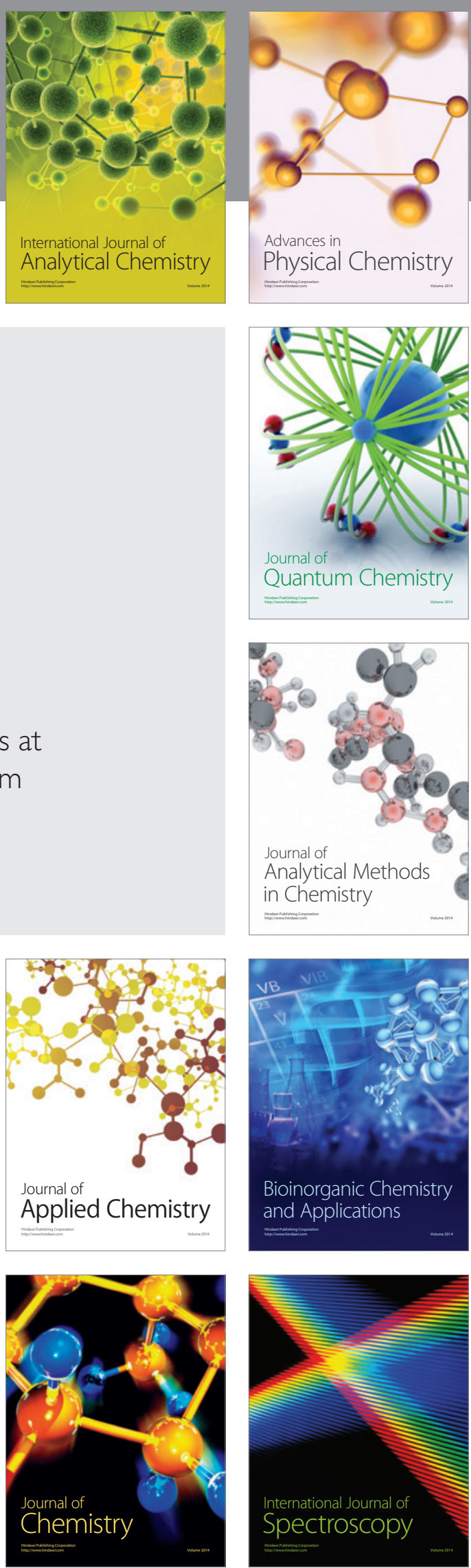